DOI: 10.17516/1997-1397-2020-13-6-774-780

УДК 512.54

\title{
Patterns of Magnetohydrodynamic Flow in the Bent Channel
}

\author{
Alexander V.Proskurin* \\ Altai State Technical University \\ Barnaul, Russian Federation \\ Anatoly M. Sagalakov ${ }^{\dagger}$ \\ Altai State University \\ Barnaul, Russian Federation
}

Received 26.07.2020, received in revised form 05.08.2020, accepted 20.09.2020

\begin{abstract}
The article considers the flow patterns of an electrically-conductive fluid in a 90 degree bend. The magnetic field is directed parallel to the outlet branch of the bend. Magnetohydrodynamic equations in terms of the small magnetic Reynolds numbers approach and the spectral-element method were used. The flow patterns were studied at different values of the Reynolds and the Hartmann numbers, and with regard to different values of the bent radus. A reverse flow was found in the outlet branch of the channel.
\end{abstract}

Keywords: magnetohydrodynamics, channel flows, spectral-element method.

Citation: A.V. Proskurin, A.M. Sagalakov, Patterns of Magnetohydrodynamic Flow in the Bent Channel, J. Sib. Fed. Univ. Math. Phys., 2020, 13(6), 774-780. DOI: 10.17516/1997-1397-2020-13-6-774780 .

\section{Introduction}

The phenomenon of a magnetic field interaction with fluid is observed in nature, and is widely used in industry. Plasma in some cases can be considered as a viscous electrically-conducting fluid. It is intended to use liquid metals for cooling advanced nuclear and thermonuclear reactors, and in large batteries that are designed to buffer energy from wind- and solar-power plants. For the design of such devices, it is important to understand the interaction mechanism of the folded flow of the electrically conducting fluid with the magnetic field. In the cases of a jet and a single vortex influenced by a transverse magnetic field, this issue was considered in $[1,2]$. In these papers it was found that a vortex in a uniform transverse magnetic field can generate secondary vortices that rotate in the reverse direction. In [3], it is described that similar phenomena can be observed in a bent channel in a vertical magnetic field in that a reverse flow was observed in the inlet branch. In this paper, we study conditions for the origin of the reverse flow in the bend in the presence of a horizontal magnetic field.

\section{Equations and numeric method}

Consider the flow in a bent channel as shown in Fig. 1. The length of the input and output branches are indicated as $L_{1}$ and $L_{2}$ respectively. The channel width is $2 d$, and the bend radius is $R$. The flow of the electrically-conducting viscous fluid occurs under a constant pressure gradient between the "inflow" and "outflow". The state Hartmann flow with the maximal velocity $V_{0}$

\footnotetext{
*k210@list.ru https://orcid.org/0000-0002-4485-2120

†amsagalakov@mail.ru

(C) Siberian Federal University. All rights reserved
} 
forms in the inlet branch. The Reynolds number is

$$
R e=\frac{V_{0} d}{\nu}
$$

where $\nu$ is the viscosity. The Hatrmann number is

$$
H a=d B_{0} \sqrt{\frac{\sigma}{\rho \nu}},
$$

where $B_{0}$ is the magnetic field, $\sigma$ is the electrical conductivity, and $\rho$ is the density of the fluid.

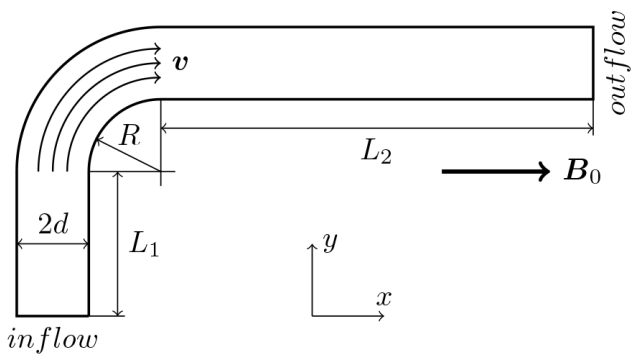

Fig. 1. The bent channel

The problem is considered under the assumption that the magnetic field generated by the movement of the fluid does not affect the flow. This small magnetic Reynolds number approach is suitable for most engineering applications [4]. It is now possible to write the equations in the following form:

$$
\begin{aligned}
\frac{\partial \boldsymbol{v}}{\partial t}+(\boldsymbol{v} \cdot \nabla) \boldsymbol{v} & =-\frac{1}{\rho} \nabla p+\nu \Delta \boldsymbol{v}+\boldsymbol{F}\left(\boldsymbol{v}, \boldsymbol{B}_{0}\right), \\
\nabla \cdot \boldsymbol{v} & =0,
\end{aligned}
$$

where $\boldsymbol{v}$ is the fluid velocity, $p$ is the pressure, and $\boldsymbol{F}$ is the magnetic force.

Omh's law is

$$
\boldsymbol{j}=\sigma\left(-\nabla \varphi+\boldsymbol{v} \times \boldsymbol{B}_{0}\right)
$$

where $\boldsymbol{j}$ is the electric current density, $\varphi$ is the electric potential. A condition $\nabla \cdot \boldsymbol{j}=0$ for the electric current leads to

$$
\Delta \varphi=\nabla \cdot\left(\boldsymbol{v} \times \boldsymbol{B}_{0}\right) .
$$

Using Reynolds (1) and Hartmann (2) numbers, equations (3) can be written in a nondimensional form

$$
\begin{gathered}
\frac{\partial \boldsymbol{v}}{\partial t}+(\boldsymbol{v} \cdot \nabla) \boldsymbol{v}=-\nabla p+\frac{1}{R e} \Delta \boldsymbol{v}+\frac{H a^{2}}{R e}\left(-\nabla \varphi+\boldsymbol{v} \times \boldsymbol{B}_{0}\right) \times \boldsymbol{B}_{0} \\
\nabla \cdot \boldsymbol{v}=0 \\
\Delta \varphi=\nabla \cdot\left(\boldsymbol{v} \times \boldsymbol{B}_{0}\right) .
\end{gathered}
$$

As the flow is two-dimensional, the flow fields do not depend upon the coordinate $z$, and $v_{z}=0$. This approximation leads to the simplified form $\Delta \varphi=0$ of the electric potential equation, and hence $\varphi \equiv 0$. Consequently, the magnetic forces will take the form $\boldsymbol{F}\left(\boldsymbol{v}, \boldsymbol{B}_{0}\right)=\frac{H a^{2}}{R e}(\boldsymbol{v} \times$ $\left.\boldsymbol{B}_{0}\right) \times \boldsymbol{B}_{0}$ and the electric potential is excluded from the equations (6). 
Boundary conditions $\boldsymbol{v}=0$ are set on the channel walls. At the inflow, the Hartmann flow is established

$$
v_{y}(x)=\frac{\cosh (H a)-\cosh (H a \cdot x)}{\cosh (H a)-1} .
$$

At the outflow, the velocity satisfies the condition

$$
\frac{\partial \boldsymbol{v}}{\partial \boldsymbol{n}}=0
$$

The spectral-element method and the computer program described earlier in [5] were used for calculations. The mesh is shown in Fig. 2. The state flow was determined by integrating equations over time until constant values of at least eight digits were established at test points. The Fig. 3 shows some of these points, marked with the letters $A, B, C$. The Tab. 1 contains the velocity values at these points when increasing the approximation order $p$ from 5 to 12 . The convergence has been achieved to at least five significant digits. A similar analysis with different grids was performed in [3].

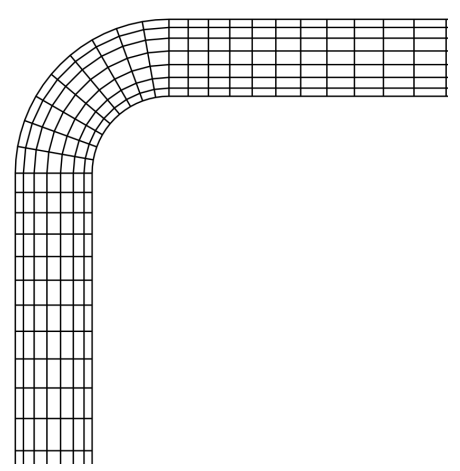

Fig. 2. The mesh

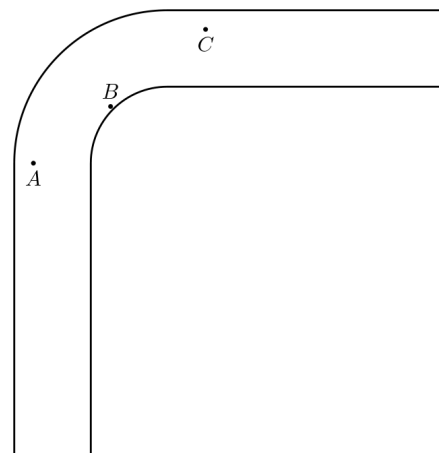

Fig. 3. The test points

Table 1. Convergence at the test points

\begin{tabular}{|c|c|c|c|c|}
\hline$p$ & $A, v_{y}$ & $B, v_{y}$ & $C, v_{x}$ & $C, v_{y}$ \\
\hline 5 & 0.98470321 & 0.8643492 & -0.03093629 & -0.00133713 \\
7 & 0.98465574 & 0.8639382 & -0.03093710 & -0.00131847 \\
10 & 0.98465842 & 0.8638807 & -0.03093676 & -0.00132150 \\
12 & 0.98465844 & 0.8638864 & -0.03093673 & -0.00132158 \\
\hline
\end{tabular}

\section{Results and discussion}

Primarily, the flow at small Reynolds numbers was considered. Fig. 4 shows streamlines in the outlet branch at $R e=0.1$ and $H a=10$ (a), $H a=35$ (b), $H a=100$ (c), and $H a=300$ (d). At $H a=10$, the streamlines are parallel. At $H a=35$, a small vortex is observed near the outer wall after the bend. At $H a=100$, a reverse flow is observed near the outer wall of the outlet branch. When the Hartmann number increases to $H a=300$, the reverse flow area shifts to the center and a return jet is formed near the channel axis. The corresponding velocity profiles in the middle of the length of the outlet branch are shown in Figs. 5 and 6. Fig. 6 shows the velocity at a large scale by a dashed line. The magnitude of the reverse flow has a perceptible value by comparison to the velocity scale $V_{0}$. 
(a)

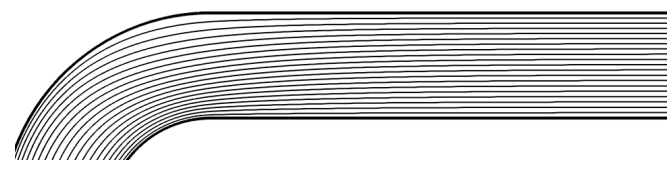

(b)

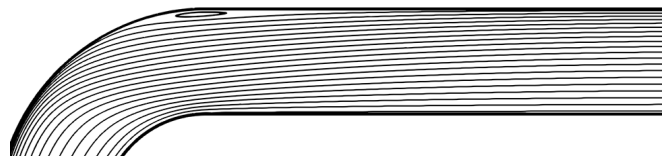

(c)

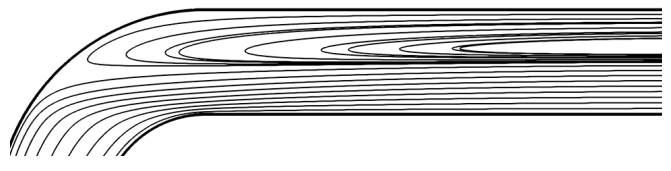

(d)

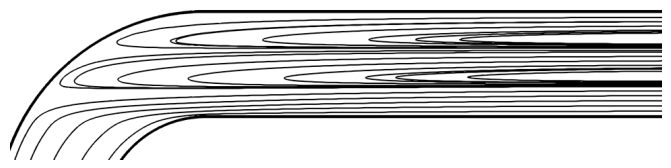

Fig. 4. Streamlines at $R e=0.1, R=2: H a=10$ (a), $H a=35$ (b), $H a=100$ (c), $H a=300$ (d)

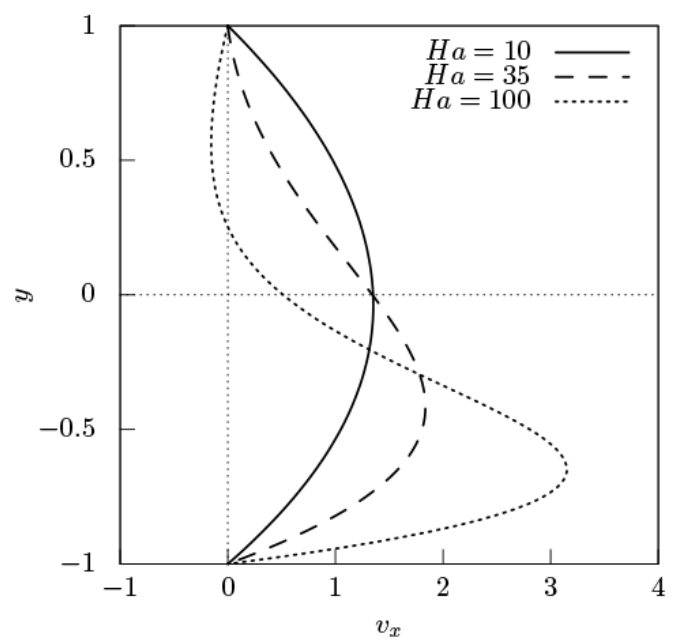

Fig. 5. Velocity $v_{x}$ in the outlet branch at $R=2, R e=0.1: H a=10, H a=35$, $H a=100$.

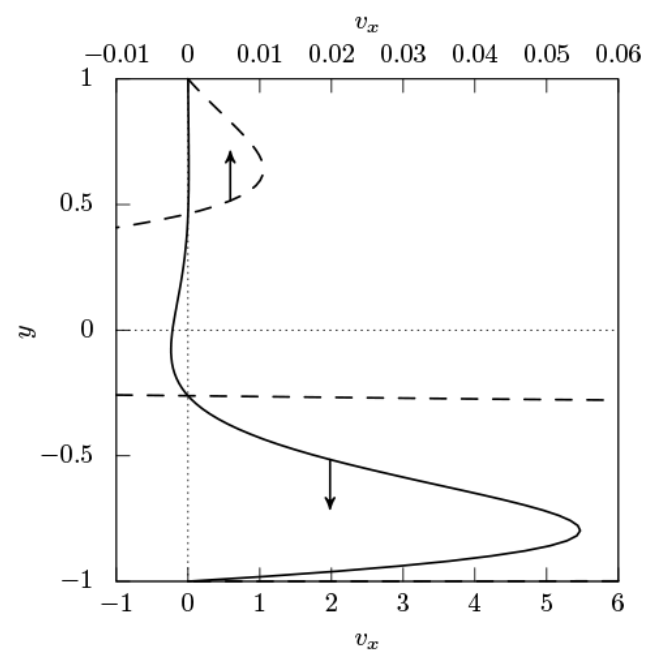

Fig. 6. Velocity $v_{x}$ in the outlet branch at $R=2, R e=0.1, H a=300$. The dashed line shows the plot at the large scale.

Fig. 5 shows that for $H a=10$ the velocity profile is symmetrical. When the magnetic field increases, the velocity maximum drifts to the inner wall. To move to the upper part of the outlet branch, the fluid would have to flow across the magnetic field. With regard to this direction, the magnetic force supresses the movement of the fluid. In the inlet branch, this magnetic braking is compensated for by the pressure gradient, but there are no forces that would cause a vertical movement in the outlet pipe. Such forces exist only in the bend, where the velocity distribution is formed due to the action of the inertia forces, the magnetic forces, and the gradient of the pressure field. In Fig. 4 (c), one can see that the streamlines from the input branch take up only 
the lower half of the outlet branch. In the upper half, the fluid is dragged by viscous forces, and the flowing forward volume is compensated for by the reverse flow from the outlet of the channel.

The mechanics of motion in a bent channel is similar to that of a free vortex in the transverse field, for which analytical solutions and estimates were obtained in [2]. The origin of the pair of reverse vortices was described in $[1,2]$ and is analogous to the origin of the reverse flow.

Fig. 7 shows the dependencies of the critical Hartmann number $H a_{*}$ from the Reynolds number. The critical Hartmann number is the number at which the reverse flow occurs, initially in the form of a small vortex as shown in Fig. 4(b). The bend radii were considered equal to $R=1,2$ and 3. For the $R e \rightarrow 0$, the dependencies $H a_{*}(R e)$ have horizontal asymptotes, that is, the occurrence of the reverse flow does not depend on the Reynolds number. At $10<R e<100$, these dependencies have minima. For $R e>100$, the curves $H a_{*}(R e)$ increases.

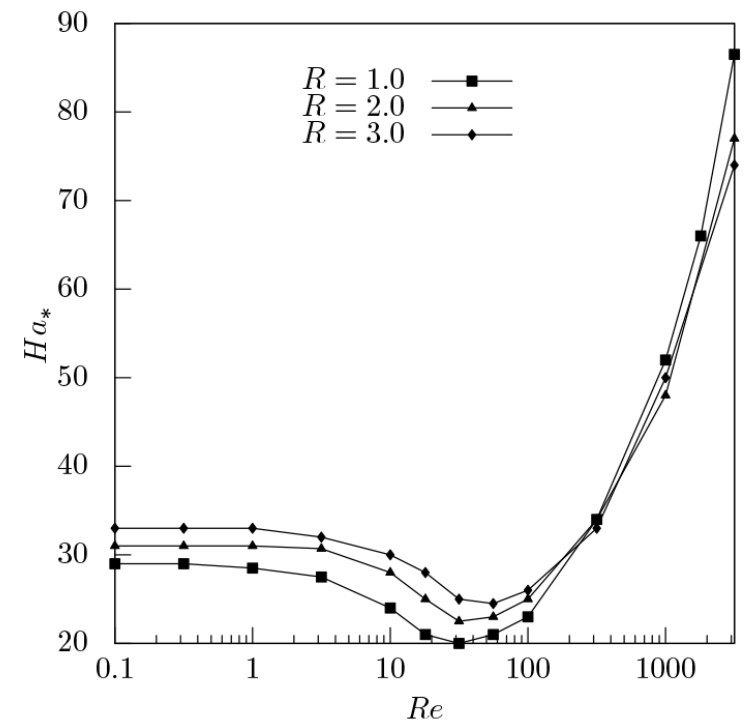

Fig. 7. Reverse flow diagram for $R=1,2,3$

Fig. 8 shows the streamlines of the reverse flow at $R e=1000: H a=10(\mathrm{a}), H a=65$ (b), $H a=100(\mathrm{c})$. At $H a=10$, a vortex is observed near the inner wall of the channel straightway after the bend. At $\mathrm{Ha}=65$, a vortex exists at the outer wall in the outlet branch. When $H a=100$, a reverse flow is observed. At the Reynolds number $R e=1000$ it was not possible to obtain a flow without vortices, as shown in Fig. $4(\mathrm{a})$ : at the smallest Hartmann numbers the flow has the form as in Fig. 8 (a). Also, due to instability, it was not possible to obtain a state reverse jet at $H a=300$.

\section{Conclusion}

This paper describes the flow of a viscous electrically-conducting fluid in a bent channel. The magnetic field is directed parallel to the outlet branch. The occurrence of the reverse flow in the form of the near-wall flow and the near-axial jet is presented, including the data for several bend radii. The obtained results are interesting with regard to the design of magnetohydrodynamic devices such as liquid metal blankets for thermonuclear reactors, given that they have a large number of bent channels. The suppression of rotational motion by a magnetic field should have a strong effect on their hydraulic characteristics. At the same time, the complete mathematical modeling of flows in engineering devices is currently a very expensive task. A solution of model 
(a)

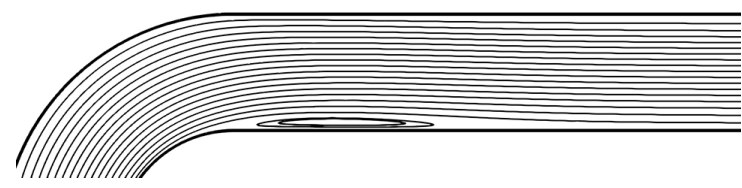

(b)

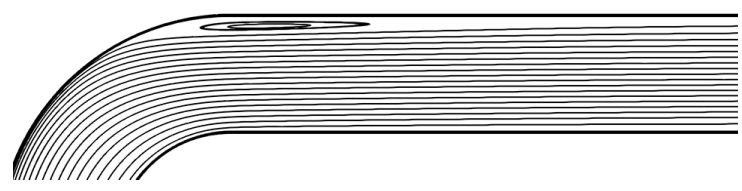

(c)

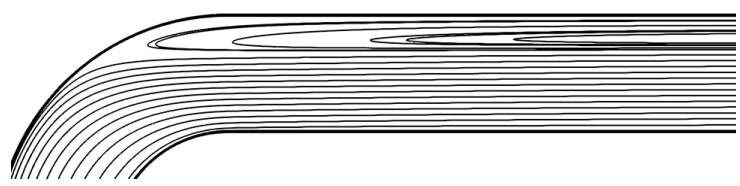

Fig. 8. Streamlines at $R e=1000, R=2: H a=10$ (a), $H a=65$ (b), $H a=100$ (c)

problems, and the identification of general laws of magnetohydrodynamic flows in bends, would have a great influence on the design of blankets and the interpretation of experimental results.

\section{References}

[1] P.A.Davidson, Magnetic damping of jets and vortices, Journal of Fluid Mechanics, 299(1995), 153-186. DOI: 10.1017/S0022112095003466

[2] P.A.Davidson, The role of angular momentum in the magnetic damping of turbulence, Journal of fluid mechanics, 336(1997), 123-150. DOI: 10.1017/S002211209600465X

[3] A.V.Proskurin and Anatoly M Sagalakov, An origin of magnetohydrodynamic reverse flow in 90 degree bends, Physics of Fluids, 30(2018), no. 8, 081701. DOI: 10.1063/1.5046328

[4] D.Lee, H.Choi, Magnetohydrodynamic turbulent flow in a channel at low magnetic Reynolds number, Journal of Fluid Mechanics, 439(2001), 367-394.

DOI: $10.1017 /$ S0022112001004621

[5] A.V.Proskurin, A.M.Sagalakov, Spectral/hp element MHD solver, Magnetohydrodynamics, 54(2018), no. 4, 361-372. 


\title{
Режимы магнитогидродинамического течения в изогнутом канале
}

\author{
Александр В. Проскурин \\ Алтайский государственный технический университет \\ Барнаул, Российская Федерация \\ Анатолий М. Сагалаков \\ Алтайский государственный университет \\ Барнаул, Российская Федерация
}

\begin{abstract}
Аннотация. В работе рассмотрены режимы течения электропроводящей жидкости в изогнутом на 90 градусов канале. Магнитное поле направлено параллельно выходному патрубку канала. Использовались уравнения магнитной гидродинамики в приближении малых магнитных чисел Рейнольдса и спектрально-элементный метод. Паттерны течения изучены при разных значениях чисел Рейнольдса и Гартмана, разных радиусах изгиба канала. Обнаружено возникновение противотечения в выходной части канала.
\end{abstract}

Ключевые слова: магнитная гидродинамика, течения в каналах, спектрально-элементный метод 